\title{
Páramos Neotropicales como unidades biogeográficas
}

\author{
Carlos Javier Diaz Acevedo ${ }^{1}$, Leidy Viviana Romero Alarcon ${ }^{1}$ \& \\ Daniel Rafael Miranda-Esquivel ${ }^{1 *}$ \\ 1. Laboratorio de Sistemática y Biogeografía, Escuela de Biología, Universidad Industrial de Santander, Bucaramanga, \\ Santander,Colombia; cjda1396@gmail.com, alarconvv@gmail.com,dmiranda@uis.edu.co \\ * Correspondencia
}

Recibido 16-X-2019. Corregido 25-II-2020. Aceptado 10-III-2020.

\begin{abstract}
Neotropical páramos as biogeographic units. Introduction: Páramo has been defined from various points of view, which take into account different factors that are easy to recognize or measure, nevertheless at the biogeographic level it has been evaluated with criticized methods used to identify historical units. The analysis of endemicity, despite its importance and wide recognition, has not been used as a tool to evaluate Páramo. Objective: Determine whether the neotropical Páramo is one or several biogeographic units. Methods: We included distributional records from Aves, Amphibia, Mammalia, Reptilia, Marchantiophyta, and Spermatophyta. We found 7025 species with 193250 suitable occurrences obtained from the GBIF. We used each taxonomic group as an independent partition or as a component of a larger partition, such as total plants (Plants-T: Marchantiophyta + Spermatophyta), or total animals (Animals-T: Aves + Amphibia + Mammalia + Reptilia), or total evidence (Plants-T + Animals-T). In order to identify areas of endemism, we used the optimality criterion (NDM/VNDM) with grids of $0.5^{\circ}$ or $0.25^{\circ}$. We calculated the intersection among polygons of previous definitions and the areas recovered in our analyses. Results: Both grid sizes, $0.25^{\circ}$ and $0.5^{\circ}$, identified areas of endemism in different sectors along the Andean and Central American cordilleras, but only the $0.25^{\circ}$ size allowed us to recognize areas/sectors with a higher resolution. We recovered eight areas, which were considered as subprovinces (Santa Marta-Perijá, Mérida, Santanderes-Boyacá, Cundinamarca, Central-Western Cordillera, Northern Ecuador, Central-South Ecuador, and Talamanca). These areas were between 4 and $66 \%$ consistent with previous definitions. Conclusions: Páramo has been considered a single biogeographic unit, however, given our analyses we identified it as a unit composed of eight biogeographic subprovinces, which is consistent with some published studies.
\end{abstract}

Key words: area of endemism, NDM/VNDM, endemicity analysis, biogeographic subprovince, Páramo.

Diaz Acevedo, C.J., Romero Alarcon, L.V., \& Miranda-Esquivel, D.R. (2020). Páramos Neotropicales como unidades biogeográficas. Revista de Biología Tropical, 68(2), 503-516.

La región de alta montaña de la zona Andina Neotropical representa uno de los ecosistemas más especiales del planeta (Madriñán, Cortés, \& Richardson, 2013). El Páramo, siendo un área tan pequeña comparada con otros ecosistemas como el Bosque Tropical, alberga gran cantidad de especies de las cuales una amplia proporción corresponde a especies únicas (Gonzalez-Duran, 2016). El Páramo es considerado como centro de diversificación para diferentes grupos taxonómicos (Madriñán et al., 2013; Rangel, 2000; Vuilleumier, \& Monasterio, 1986), además de ser reconocido por su alta complejidad geológica; la cual está ligada directamente a la orogénesis andina del Bloque Norte de los Andes (Buytaert, Iniguez, $\&$ De Bievre, 2007). Sin embargo, no solo sus particularidades históricas y biológicas lo han 
hecho atractivo para las investigaciones en diferentes ramas, sino también su fragilidad a los cambios abruptos del ambiente, su papel en el desarrollo cultural de las poblaciones (Morales-Rivas et al., 2007) y su capacidad de actuar como reservorio de agua (Cortés-Duque \& Sarmiento, 2017).

La palabra páramo proviene del latín parămus, que significa "terreno yermo, raso $y$ desabrigado, lugar frio y desamparado" (RAE, 2014); este es un concepto europeo, aplicado por los exploradores españoles a un bioma tropical, debido a su semejanza con las llanuras áridas de la península Ibérica (Luteyn, 1999). Actualmente, Páramo es definido como un ecosistema húmedo tropical ubicado a partir del límite superior del bosque con vegetación propia, de tipo herbácea y arbustiva (Hofstede, Segarra, \& Vásconez, 2003). Los Páramos se distribuyen en las regiones de alta montaña de Ecuador, Colombia, en Venezuela sobre la región de Mérida, Panamá y Costa Rica (Hofstede et al., 2014; Luteyn, 1999). En el Bloque Norte de los Andes, su origen data del Plioceno, época en la cual las cordilleras alcanzaron su altitud actual (Van der Hammen, 1997) y su vegetación ya era propia del Páramo contemporáneo (Wijninga, 1996), pero con su límite altitudinal por debajo (1700 m.s.n.m.) del límite actual (2 800 - 3000 m.s.n.m.) (Flantua \& Hooghiemstra, 2018; Van der Hammen \& Gonzáles, 1963). No obstante, dicho límite ha sido dinámico (Flantua, O'dea, Onstein, Giraldo, \& Hooghiemstra, 2019), ya que durante el Cuaternario (2.4 Ma) y hasta el presente, han ocurrido entre 22 a 24 ciclos glacial-interglacial (Diazgranados \& Barber, 2017), permitiendo que islas de páramos se conectaran y se separaran durante estos periodos de tiempo, impulsando el proceso de especiación y de generación de especies endémicas (Benham, Cuervo, McGuire, \& Witt, 2015; Jørgensen \& Ulloa, 1994; Pouchon et al., 2018; Quintero, Ribas, \& Cracraft, 2012; Van der Hammen \& Cleef, 1986).

Son diversos los acercamientos que se han tenido en cuenta para definir Páramo, lo cual ha resultado en una plétora de delimitaciones que son, o no, parcialmente congruentes entre sí.
Dichas definiciones están sujetas al objeto de estudio del investigador y solo tienen en cuenta factores de fácil reconocimiento y/o medición. Por ejemplo, Cuatrecasas (1958) afirma que los páramos son regiones de gran extensión ubicadas arriba del Bosque Andino entre 3800 m.s.n.m hasta 4700 m.s.n.m, dividiéndose en Subpáramo, Páramo y Superpáramo de acuerdo a sus características florísticas. Otros autores han considerado en conjunto factores bióticos $\mathrm{y}$ abióticos, tales como la altitud y algunas comunidades de plantas; siendo estos últimos los más utilizados para delimitar esta región en términos ecológicos (Guhl, 1982; Monasterio, 1980; Rangel, 2000; Sarmiento et al., 2013; Vargas \& Pedraza, 2004).

Morrone (2017) plantea Páramo como “... of the Andean cordillera of Venezuela, Colombia, and Ecuador, from the upper forest line at 3000 - 3500 meters upwards and below the permanent snowline at ca. 5000 meters. This high-elevation biome has been influenced by glaciations, being the landscape irregular, from very rough to flat and stretches from 3000 meters to the perennial snowline at altitudes of 4800 - 5000 meters" (p. 218). Este es uno de los primeros acercamientos que plantea Páramo como una unidad histórica, y en definirlo formalmente en términos de biogeografía (Morrone 2001, 2014, 2017). Sin embargo, dicha propuesta se basó en el compendio del conocimiento previo de múltiples autores y no en una hipótesis a testear y replicable bajo métodos cuantitativos. Otros trabajos han evaluado Páramo a nivel biogeográfico (Jiménez-Rivillas, García, Quijano-Abril, Daza, \& Morrone, 2018), sin embargo se basan en métodos usualmente criticados, los cuales requieren definir áreas a priori y violan la premisa de no jerarquía en la definición de área de endemismo en biogeografía histórica (Szumik, Cuezzo, Goloboff, \& Chalup, 2002), o simplemente no presentan una delimitación clara de Páramo, ni se basan en la definición de áreas de endemismo (Hazzi, Moreno, Ortiz-Movliav, \& Palacio, 2018). Adicionalmente en estos trabajos, las zonas de alta montaña de Costa Rica y el norte de Perú siempre son excluidos de los análisis, 
probablemente, porque no son parte de la definición de Van der Hammen (1974), trabajo en el cual se han basado la mayoría de estudios de Páramo. Por tanto, un reanálisis, bajo métodos de optimización, de toda la extensión del área que históricamente se ha considerado como Páramo, es relevante para poder reconocerla como unidad biogeográfica.

Un área de endemismo se define como "the congruent distributional limits of two or more species" (Platnick, 1991). Sus límites están marcados generalmente por eventos vicariantes y podrían ser difusos por efecto de expansión del rango de distribución de las especies y su dinamismo a través del tiempo (Zunino \& Zullini, 2003). Para el reconocimiento de unidades biogeográficas se ha implementado diferentes algoritmos como: el Análisis de Parsimonia de Endemismos (PAE) (Rosen \& Smith, 1988), el Análisis de Parsimonia de Endemismos para cuadrículas (PAE) (Morrone, 1994), el criterio para el reconocimiento de áreas de endemismo (Harold \& Mooi, 1994), el criterio de optimización para evaluar métodos de endemismo (Linder, 2001), el Análisis de Endemicidad (AE) (Szumik et al., 2002; Szumik \& Goloboff, 2004), el Análisis de Co-presencia Significante de Taxones (Sigcot) (Mast \& Nyffeler, 2003), Sigcot con PAE (Giokas \& Sfenthourakis, 2008), el Análisis de Endemismos de Áreas Anidadas (NAEA) (Deo \& DeSalle, 2006), el Método de Análisis de Redes (NAM) (Dos Santos, Fernández, Cuezzo, \& Dominguez, 2008) y la Interpolación Geográfica de Endemismo (GIE) (Oliveira, Brescovit, \& Santos, 2015).

De los anteriores, el análisis de endemicidad propuesto por Szumik et al. (2002) y Szumik y Goloboff (2004), ha sido el más aceptado para el análisis de endemismo en los últimos años, dadas sus ventajas sobre los otros métodos (Alvez-Valles et al., 2018; Martínez-Hernández et al., 2015; Sánchez, Garcia-Cruz, Espejo-Serna, \& Lopez-Ortega, 2019; Zhuang et al., 2018). Este reconocimiento se debe a que tiene en cuenta de manera explícita el componente espacial, además de identificar áreas superpuestas, núcleos y límites difusos (Aagesen, Szumik, \& Goloboff, 2013; Casagranda, Taher, \& Szumik, 2012; MartínezHernández et al., 2015; Szumik et al., 2002). Pese a su importancia y amplio reconocimiento en la comunidad, hasta el momento no se ha utilizado como herramienta para evaluar exclusivamente las zonas de alta montaña del Bloque Norte de los Andes, Mérida y Talamanca, y es así que surge la idea de determinar si los páramos neotropicales son una o varias unidades biogeográficas.

\section{MATERIALES Y MÉTODOS}

Datos: A partir de listas de referencia, como Luteyn (1999) o Rangel (2000), identificamos las clases y divisiones taxonómicas con mayor número de especies presentes en lo que hasta el momento se ha definido como Páramo. Teniendo en cuenta los límites de distribución de páramo $\left(11^{\circ} \mathrm{N}, 8^{\circ} \mathrm{S}, 84^{\circ} \mathrm{W}, 70^{\circ} \mathrm{W}\right)$ propuestos en Luteyn (1999) descargamos los datos disponibles de los diferentes taxones seleccionados (Aves, Amphibia, Mammalia, Reptilia, Marchantiophyta y Spermatophyta) desde la plataforma de información de biodiversidad global GBIF. Además, revisamos la taxonomía y validez del nombre de cada especie en bases de datos como The Integrated Taxonomic Information System [ITIS] (Shaw, 2004) y Tropicos (Tropicos, 2018). Posteriormente, de acuerdo a las recomendaciones de Chapman (2005), realizamos una limpieza exhaustiva de datos, siguiendo el protocolo propuesto por Romero-Alarcon (2016) e implementado en el paquete biogeoclean (Romero-Alarcon \& Miranda-Esquivel, 2015) de $R$ (R Core Team, 2019). Primero, obtuvimos una lista de especies con distribución sobre los 1900 m.s.n.m (altura mínima de distribución de los páramos), y eliminamos las especies exóticas e invasoras (IBIS (Kells \& Worswick, 1997) e ISSG (Lowe et al., 2000)). Luego, con esta nueva lista de especies, descargamos de GBIF todos los puntos georeferenciados para cada una de las especies, eliminamos puntos mal ubicados, especies ampliamente distribuidas, y especies con menos de tres presencias; para finalmente, 
obtener especies con presencias viables para nuestros análisis.

\section{Búsqueda y visualización de áreas de} endemismo: Usamos como partición cada grupo taxonómico previamente mencionado. Asimismo, estos taxones se agruparon para generar particiones adicionales como: plantas totales (Plantas-T: Marchantiophyta + Spermatophyta), animales totales (Animales-T: Aves + Amphibia + Mammalia + Reptilia) y evidencia total (Plantas- $\mathrm{T}+$ Animales- $\mathrm{T}$ ). En total en nuestro estudio usamos nueve particiones, las cuales se analizaron de manera independiente para evidenciar el aporte diferencial de cada una de ellas al patrón general.

Para identificar áreas de endemismo sensu Platnick (1991), utilizamos el criterio de optimalidad propuesto por Szumik et al. (2002) y Szumik y Goloboff (2004) e implementado en el algoritmo eNDeMism/ Visual of eNDeMism (NDM/VNDM) (Goloboff, 2005). Para ello, realizamos búsquedas heurísticas de 10 repeticiones, manteniendo áreas con dos o más especies endémicas y con índice de endemicidad mayor o igual a 2.0, además de conjuntos de subóptimos con 0.90 de ajuste mínimo. La semilla de aleatorización fue 1.0 y todas las áreas se mantuvieron independientemente del porcentaje de solapamiento entre especies únicas. Estos parámetros fueron seleccionados siguiendo el análisis de Escalante, RodríguezTapia, Szumik, Morrone, \& Rivas (2010) para la delimitación de áreas de endemismo en la región Neártica. Siguiendo las recomendaciones de Aagesen, Szumik, Zuloaga y Morrone (2009) y Szumik et al. (2012), cada partición la evaluamos independientemente bajo dos tamaños de cuadrícula diferentes $0.25^{\circ}$ y $0.5^{\circ}$; no usamos tamaños como $1^{\circ}$ o mayores ya que superan la extensión del páramo más grande a evaluar. Finalmente, resumimos la información obtenida usando el consenso estricto de similitud de especies bajo tres cortes distintos: 30,50 y $75 \%$ (Aagesen et al., 2013).

Comparación de áreas con propuestas de otros autores: Construimos un vector espacial tipo polígono con cada una de las definiciones previas de Páramos: Cuatrecasas (1958), Jiménez-Rivillas et al. (2018), Monasterio (1980), Morrone (2017), Rangel (2000), Van der Hammen (1997); y Vargas y Pedraza (2004). Cada polígono fue generado extrayendo las curvas de nivel a partir de un modelo de elevación digital (DEM, por sus siglas en inglés) y proyectando estas como mapas vectoriales con ayuda de QuantumGis (QGIS Development Team, 2019). Para comparar numéricamente las definiciones previas con los consensos obtenidos, calculamos el área de sobreposición entre ellas usando la función gArea del paquete de análisis espacial Rgeos (Bivand, Rundel, Pebesma, Stuetz, \& Hufthammer, 2016). El valor de la sobreposición para cada una de las comparaciones se escaló sobre el área total de cada área de endemismo encontrada.

\section{RESULTADOS}

Después de realizar la actualización taxonómica $\mathrm{y}$ depurar las presencias obtenidas desde GBIF, encontramos 7025 especies (570 Amphibia, 119 Reptilia, 272 Aves, 70 Mammalia, 189 Marchantiophyta y 5725 Spermatophyta) con 193250 presencias viables para nuestros análisis. De estas, 954 especies (116 Amphibia, 30 Aves, 8 Mammalia, 29 Reptilia, 5 Marchantiophyta y 766 Spermatophyta) soportaron al menos un área del total de las áreas de endemismo recuperadas bajo el análisis de evidencia total (Apéndice Digital).

Los diferentes tamaños de cuadrícula nos permitieron encontrar grupos de áreas similares, sin embargo, con el tamaño de $0.25^{\circ}$ los identificamos con mayor resolución. Lo mismo sucedió con los cortes de consenso; aunque los tres $(30,50$ y $75 \%)$ fueron aplicados para resumir cada análisis, el corte de $75 \%$ proporcionó la mejor interpretación de los resultados; además, con este valor (75\%) diferenciamos áreas de evidencia total soportadas por especies pertenecientes a una misma clase o división taxonómica. Cuando comparamos los resultados obtenidos con ambos tamaños de cuadrícula, el $99.4 \%$ de las áreas encontradas con tamaño de $0.25^{\circ}$ estuvieron contenidas en las encontradas 
con tamaño de $0.5^{\circ}$. Así mismo, cuando resumimos los resultados en cada análisis, todos los tipos de corte del consenso estricto recuperaron los mismos conjuntos de áreas, pero cada uno con número diferente de áreas consenso.

El número de arreglos iniciales fue diferencial entre tamaños de cuadrícula; por ejemplo, el análisis de la partición de evidencia total para $0.25^{\circ}$ generó 445 arreglos, en contraste a los 1040 arreglos encontrados bajo $0.5^{\circ}$. De la misma forma, encontramos 281 áreas consenso al resumir los resultados de $0.25^{\circ}$, comparadas a las 541 encontradas para $0.5^{\circ}$, ambos, bajo un corte de $75 \%$. Esto mismo ocurrió con otras particiones como Animales-T con 100 arreglos iniciales y 58 áreas consenso, o Plantas-T con 328 arreglos iniciales y 208 áreas consenso, ambos bajo un corte de $75 \%$ (Tabla 1). Por tanto, el tamaño de cuadrícula influyó directamente en la cantidad de arreglos iniciales y en el número de áreas consenso en todos los análisis realizados. Pese a este comportamiento, el patrón final o sectores del mismo fueron constantemente recuperados entre todas las particiones.

Unidades biogeográficas dentro de Páramo: Nuestros análisis permitieron identificar dentro de lo que actualmente es denominado como Páramo, ocho unidades biogeográficas: Santa Marta-Perijá, Mérida, Santanderes-Boyacá, Cundinamarca, Cordillera Central-Occidental, Norte de Ecuador, Centro-Sur de Ecuador

TABLA 1

Número de arreglos iniciales de cada partición por tamaño de cuadrícula

\begin{tabular}{lcc}
\multicolumn{1}{c}{ Partición } & Número de arreglos iniciales \\
Mammalia & $0.25^{\circ}$ & $0.5^{\circ}$ \\
Marchantiophyta & 1 & 7 \\
Reptilia & 1 & 8 \\
Aves & 6 & 16 \\
Amphibia & 19 & 28 \\
Animales-T & 60 & 180 \\
Spermatophyta & 100 & 209 \\
Plantas-T & 352 & 889 \\
Evidencia total & 328 & 929 \\
\hline
\end{tabular}

y Talamanca (Fig. 1). Dichas unidades se asignaron al nivel de subprovincia, según la taxonomía propuesta para áreas biogeográficas (Ebach, Morrone, Parenti, \& Parenti, 2008; Escalante, 2009). Estas áreas fueron comparables a la zonificación propuesta por Jiménez-Rivillas et al. (2018), trabajo del cual extrajimos la nomenclatura para asignar los nombres de nuestras subprovincias.

En términos de particiones, Plantas-T (Fig. 2A) y Animales-T (Fig. 2B) identificaron regiones semejantes a las subprovincias encontradas por la partición de evidencia total. Sin embargo, las regiones recuperadas de manera individual no fueron equivalentes entre ellas. Por ejemplo, Plantas-T recuperó, aunque no en su extensión completa, el patrón general de todas las subprovincias planteadas en este estudio (Fig. 1); dado principalmente por las especies de Spermatophyta (Fig. 2D) las cuales representaron el 97 $\%$ de la partición de Plantas-T, el $3 \%$ restante correspondió a especies de Marchantiophyta (partición que solo participó en la identificación de una única área) (Fig. 2C). Algunas subprovincias como Talamanca, Santa Marta-Perijá, Mérida, Cordillera Central-Occidental, Norte de Ecuador y Centro-sur de Ecuador, fueron recuperadas por la partición Animales-T (Fig. 2B), pero a diferencia de Plantas-T (Fig. 2A), cada una de las particiones por clase (Aves, Amphibia, Mammalia y Reptilia) reconoció diferentes subprovincias de manera individual (Fig. 2E, Fig. 2F, Fig. 2G, Fig. 2H). Por ejemplo, la partición de Animales-T fue la única que recuperó la subprovincia de Mérida (Fig. 2B), en comparación a las encontradas por cada clase taxonómica de manera individual.

Especies de Spermatophyta soportan el $98 \%$ de las áreas consenso encontradas en el análisis de evidencia total, mientras que especies de Amphibia soportan el $61 \%$ de las áreas. El resto de grupos aportaron en valores inferiores al índice de endemicidad de las áreas encontradas (Aves: $23 \%$, Mammalia: 14 \%, Reptilia: $13 \%$ ), siendo Marchantiophyta el grupo con menor contribución $(6 \%)$ al soporte de las áreas consenso. Es así que cada grupo taxonómico contribuyó diferencialmente 


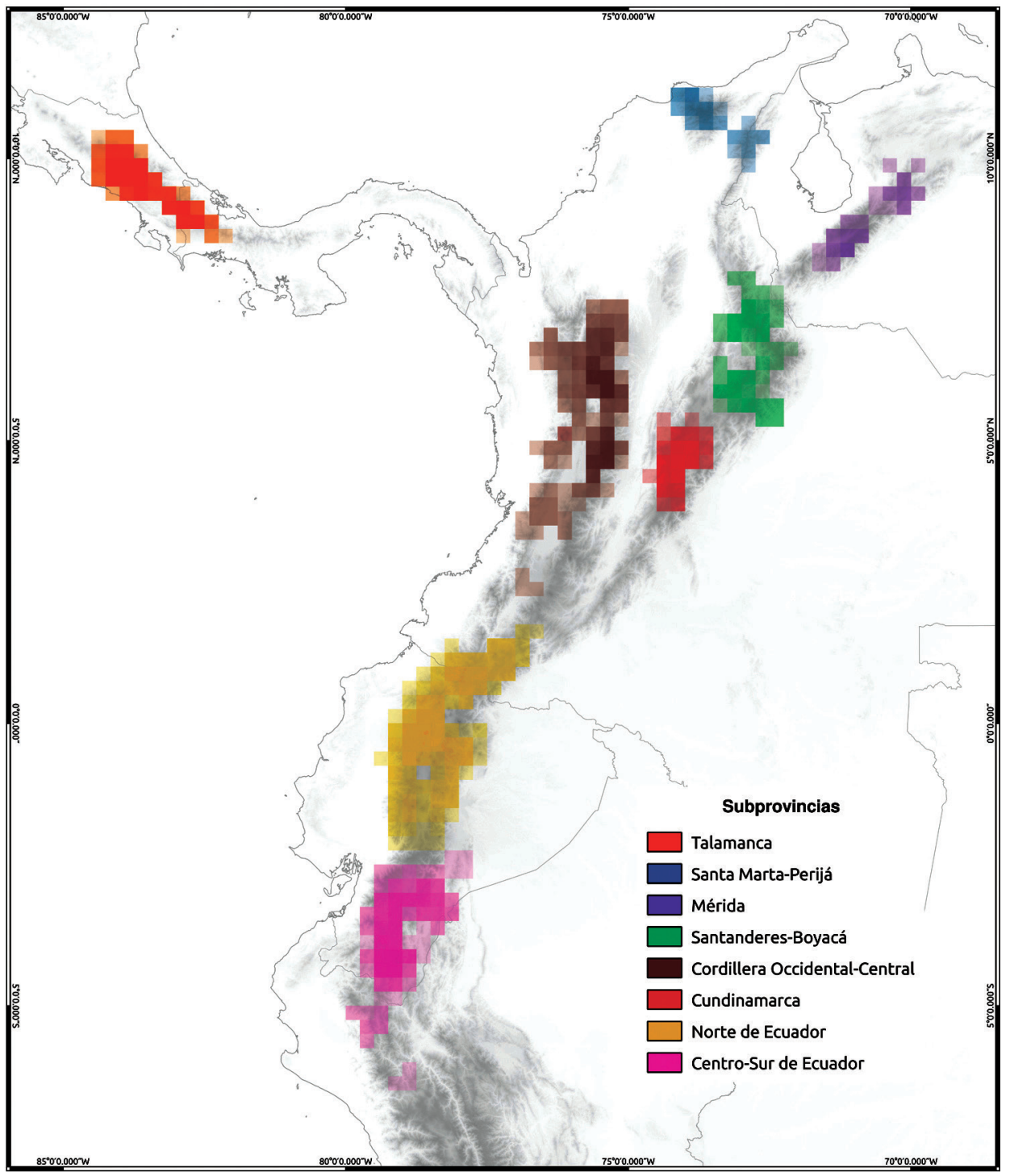

Fig. 1. Distribución geográfica de las áreas de endemismo (AE) en la provincia biogeográfica de Páramo para el tamaño de cuadrícula $0.25^{\circ}$. Consenso estricto $75 \%$, análisis de Evidencia total. La intensidad de color indica el nivel de superposición de las áreas consenso.

a la recuperación de cada una de las subprovincias, tanto de manera individual como colectiva (ver Evidencia total).

Comparación de polígonos: Nuestra propuesta comparte un gran porcentaje de similitud (66 \%) con las áreas planteadas por JiménezRivillas et al. (2018). No obstante, dos de las propuestas más aceptadas: Morrone (2017) y Cuatrecasas (1958), utilizadas frecuentemente en trabajos de ecología, biogeografía y conservación, presentaron los menores valores de similitud con nuestros resultados (16\% y $4 \%$ respectivamente). De igual forma, otras definiciones como las de Monasterio (1980), Van der Hammen (1997) y Rangel (2000) presentaron 

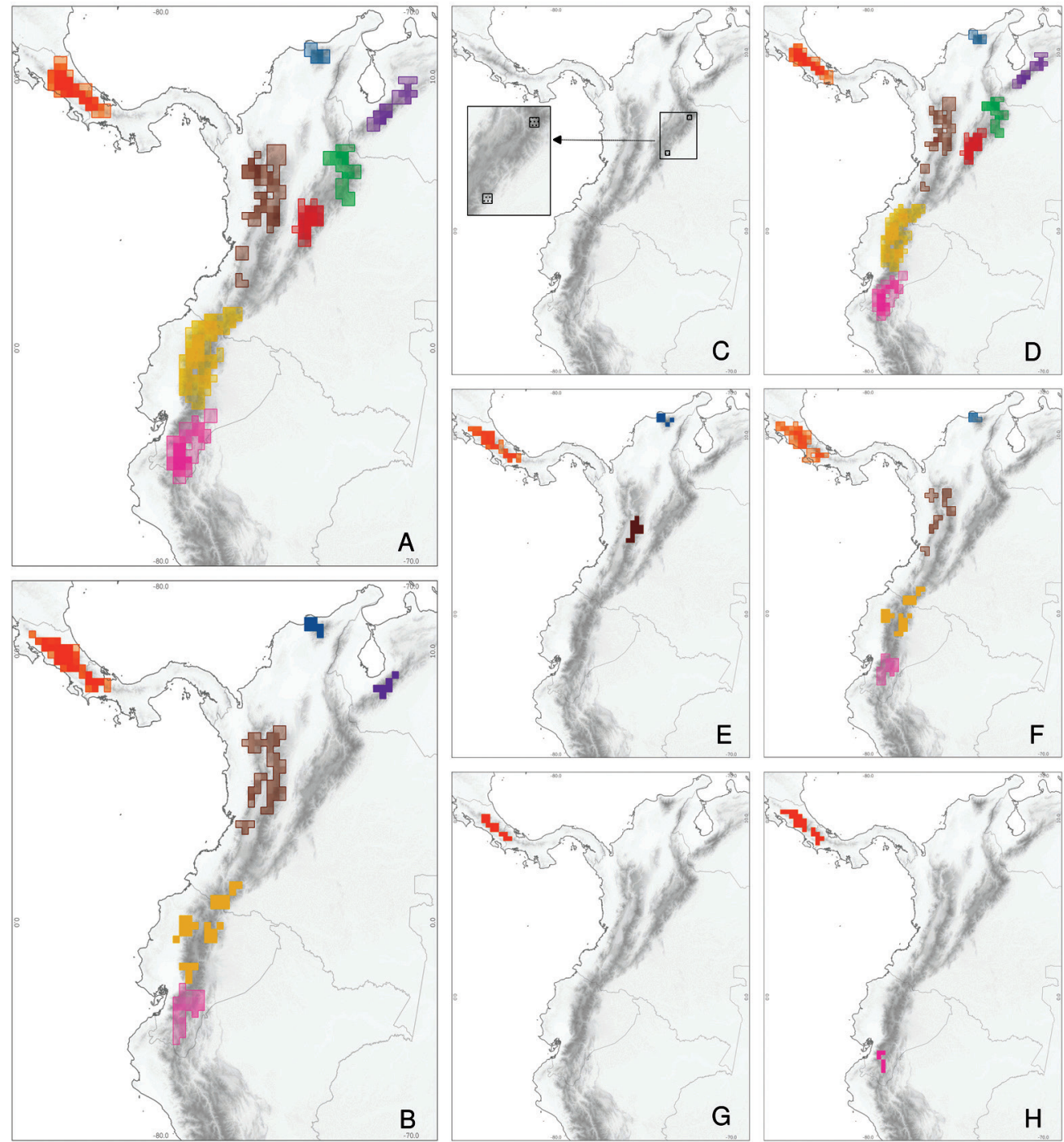

Fig. 2. Distribución geográfica de áreas de endemismo por partición (tamaño de cuadrícula $0.25^{\circ}$, consenso estricto $75 \%$ ): (A) Plantas-Total, (B) Animales-Total, (C) Marchantiophyta, (D) Spermatophyta, (E) Aves, (F) Amphibia, (G) Mammalia, (H) Reptilia. Los colores indican cada una de las subprovincias según la Fig. 1. La intensidad de color no representa el nivel de superposición de las áreas consenso, y el color del borde no representa los límites de las áreas.

similitudes bajas respecto a nuestro trabajo (20\%, $26 \%$, y $15 \%$, respectivamente).

\section{DISCUSIÓN}

Presencias a partir de bases de datos: Nuestro estudio es el primero en el cual se emplean alrededor de 7000 especies de distintos taxa para la identificación de áreas de endemismo en zonas de alta montaña, en contraste a otros estudios como Jiménez-Rivillas et al. (2018) con 4644 especies o Londoño, Cleef y Madriñán (2014) con 2158 especies.

Trabajos con amplio muestreo de presencias, obtenidos a partir de bases de datos como GBIF, implican un reto en su manipulación, 
limpieza y análisis. Generalmente dichas presencias son propensas a diferentes tipos de errores, los cuales es nuestro deber detectar y corregir para minimizar el sesgo o la incertidumbre de nuestros datos finales (Antonelli et al., 2014; Chapman, 2005). Por ello, un componente esencial de nuestro trabajo fue depurar las descargas crudas de GBIF hasta obtener registros viables para nuestro análisis. Esta actividad es central, ya que una buena práctica de limpieza de datos debería ser el primer paso del diseño experimental, para cualquier estudio biogeográfico o que utilice datos distribucionales (Zizka et al., 2019). De esta forma, la información obtenida desde plataformas como GBIF podría ser mejor aprovechada para la obtención de patrones generales de biodiversidad (García-Roselló et al., 2015; Maldonado et al., 2015).

Tamaños de cuadrícula y particiones: Aunque no existe un tamaño de cuadrícula óptimo, otros estudios sobre áreas de endemismo, como Casagranda et al. $(2009,2012)$, recomiendan el uso de cuadrículas de diferentes tamaños para visualizar distintos patrones de áreas. Con el tamaño de $0.25^{\circ}$ obtuvimos una mayor resolución de los límites de cada subprovincia, esto fue congruente con lo planteado por Szumik et al. (2012), quienes afirman que el tamaño de cuadrícula podría depender de la topografía del terreno, siendo las cuadrículas pequeñas las más adecuadas para el análisis en terrenos con pendiente alta, como por ejemplo en los Andes argentinos; idea que puede ser extendida fácilmente a nuestro trabajo.

De la misma forma que en Casagranda, Roig-Juñent y Szumik (2009) y Szumik et al. (2012), el número de arreglos iniciales fue proporcional al tamaño de cuadrícula, sin embargo, independientemente de este comportamiento, cada análisis nos permitió observar áreas equivalentes entre sí, indicándonos la consistencia del patrón general.

Trabajos como Escalante et al. (2010) proponen que el análisis de endemicidad se debe realizar con distintos taxa para que el estimador del índice de endemicidad sea robusto, con el cual se pueden hacer afirmaciones del patrón general de áreas. Por tanto, los resultados por clases taxonómicas son importantes para hacer inferencias y entender patrones individuales. Así, estos patrones individuales nos permiten ver el aporte proporcional de cada partición al patrón general. Por ejemplo, particiones como Amphibia y Spermatophyta contribuyeron en mayor proporción al patrón general, dado que contienen mayor cantidad de especies soporte. Sin embargo, esta contribución posiblemente está directamente relacionada a la riqueza de cada taxón evaluado, tal y como lo plantea Noguera-Urbano (2017).

Unidades biogeográficas: Encontramos que Páramo no es una sola unidad como se ha propuesto en estudios anteriores (Morrone, 2017); dichas unidades se identificaron parcialmente con las particiones de clases y divisiones; y el conjunto total de ellas (evidencia total) nos permitió identificar ocho subprovincias en el patrón general. Es importante aclarar que nuestras áreas de endemismo son unidades operacionales, que no son informativas sobre los límites reales de áreas, ya que nuestro objetivo implica la recuperación de núcleos/ patrones y no de delimitaciones altitudinales.

Nuestro patrón presenta mayor congruencia con la regionalización planteada por Jiménez-Rivillas et al. (2018), que con otras definiciones de Páramo; las regiones encontradas por las particiones de clase o división también son congruentes con los distritos propuestos por Jiménez-Rivillas et al. (2018), reconociendo la mayoría de estos a excepción del distrito Los Picachos. En general, la congruencia entre nuestro trabajo y Jiménez-Rivillas et al. (2018) nos muestra que subprovincias como Santa Marta-Perijá, Norte de Ecuador y Mérida fueron equivalentes en su extensión completa a los distritos de Jiménez-Rivillas et al. (2018) denominados como A (Venezuelan Páramo), D (Northern Ecuadorian Páramo) y F (Sierra Nevada). Otras subprovincias correspondieron a conjuntos de distritos, por ejemplo, la subprovincia Cordillera Central-Occidental fue equivalente a la suma de distritos G (Santa 
Inés-Sonsón), H (Paramillo del Sinú), I (Páramo de Los Farallones de Cali) y K (Páramos Quindío); de la misma forma que la subprovincia Centro-Sur de Ecuador que correspondió a la unión de los distritos B (Southern Ecuadorian Páramo) y C (Central Ecuadorian Páramo). El distrito J (Páramos de la Cordillera Oriental), en nuestro análisis lo encontramos como dos unidades independientes (Santanderes-Boyacá y Cundinamarca). En nuestro análisis el norte de Perú fue parte de la subprovincia Centro-Sur de Ecuador, la cual fue compatible con el distrito B. Finalmente, el distrito E (Los Picachos) nunca fue identificado bajo ninguna partición.

Tal similitud muestra que pese a las diferencias metodológicas (PAE en Jiménez-Rivillas et al., 2018, AE: en el presente estudio), ambas aproximaciones, independientemente, generan resultados equivalentes. Sin embargo, nuestro estudio tiene una ventaja sobre Jiménez-Rivillas et al. (2018) ya que la optimización de áreas de endemismo bajo el método de optimalidad (AE), puede ser más eficiente en la recuperación de unidades biogeográficas (Casagranda et al., 2009; Escalante, 2015). Esto, posiblemente, podría ser una de las explicaciones de que la similitud entre ambos trabajos solo fuera de un $66 \%$, a pesar de usar datos equivalentes de clases taxonómicas.

Es contraintuitivo que Morrone (1994), quien plantea el método de PAE en biogeografía y genera la regionalización del Neotrópico (Morrone, 2001, 2014, 2017), no logre reconocer las áreas identificadas en Jiménez-Rivillas et al. (2018) o en el presente estudio. Esto implica que es necesario hacer una revisión de la regionalización del Neotrópico. Un ejemplo de ello se puede encontrar en el estudio realizado por Romero-Alarcon (2016), quien evalúa, usando el método $\mathrm{AE}$, la provincia del Chocó biogeográfico propuesta por Morrone (2014), encontrando que Chocó biogeográfico no corresponde a una única región como lo plantea Morrone (2014) sino que corresponde a tres regiones distintas, similares a las propuestas por Hernández-Camacho, Hurtado, Ortiz, \& Walschburger (1992).
Cuando hablamos de Páramo en términos biogeográficos debemos referirnos a ocho subprovincias que no solo involucran las zonas de alta montaña de Colombia, Venezuela y Ecuador, sino que también deben incluir a Talamanca en Costa Rica. Esta última área ha sido considerada como Páramo en otras definiciones (Cleef \& Chaverri, 1992; Luteyn, 1999; Luteyn \& Balslev, 1992), basadas en su afinidad taxonómica con otros páramos colombianos. Por ejemplo, la presencia de algunos géneros de plantas (Myrrhidendron, Pterichis, Chaetolepis, entre otros) distribuidos en las regiones de alta montaña de Talamanca y los páramos en la Sierra Nevada de Santa Marta (Cleef \& Chaverri, 1992). Esto mismo sucede con el norte de Perú, aunque algunos autores prefieren definirla como otra región denominada como Jalca, dada su estructura florística (Brack \& Mendiola, 2000; Cabrera \& Willink, 1980; de Mera et al., 2017; Van der Hammen, 1974), en nuestro caso, la encontramos como parte de la subprovincia Centro-Sur de Ecuador.

Nuestros resultados no identifican la sectorización tipo Subpáramo, Páramo y Superpáramo propuesta por Cuatrecasas (1958), ya que $\mathrm{AE}$ no permite identificar límites altitudinales, ni utilizar datos como comunidades de plantas o factores abióticos como el clima. Así mismo, nuestros resultados tampoco son comparables con la delimitación propuesta por Morales-Rivas et al. (2007), la cual se basa en criterios biofísicos, socioculturales e integridad ecológica. Podemos afirmar que nuestro trabajo permite observar el patrón general de unidades históricas de las zonas que han sido consideradas como Páramo, las cuales podrían ser la base para la resolución de preguntas en diferentes áreas como biogeografía histórica de patrón y eventos, diversificación, macroecología y conservación.

En conclusión, independientemente del tamaño de cuadrícula, AE permite identificar patrones generales consistentes. Adicionalmente, el uso de cuadrículas pequeñas o proporcionales al área de estudio, nos permite encontrar resultados más ajustados y de alta resolución. Páramo se ha considerado como diferentes 
áreas dadas algunas características como la altitud o el tipo de vegetación. En biogeografía estas áreas se han planteado como una sola unidad, mientras que nuestro trabajo define Páramo como ocho subprovincias biogeográficas las cuales podrían ser consideradas como la base para futuros análisis.

Recomendamos revisar las regionalizaciones del Neotrópico a escalas más pequeñas de región o provincia; además de explorar tamaños de cuadrícula más pequeños dentro de cada subprovincia, e involucrar métodos que incluyan directamente la elevación, de tal forma que se puedan plantear límites altitudinales de estas regiones de alta montaña.

Declaración de ética: los autores declaran que todos están de acuerdo con esta publicación y que han hecho aportes que justifican su autoría; que no hay conflicto de interés de ningún tipo; y que han cumplido con todos los requisitos y procedimientos éticos y legales pertinentes. Todas las fuentes de financiamiento se detallan plena y claramente en la sección de agradecimientos. El respectivo documento legal firmado se encuentra en los archivos de la revista.

\section{AGRADECIMIENTOS}

A los miembros del Laboratorio de Entomología y el Laboratorio de Sistemática y Biogeografía de la Universidad Industrial de Santander, por su tiempo y valiosos aportes. DRM-E agradece a la Vicerrectoría de Investigación y Extensión de la Universidad Industrial de Santander, Colombia. Proyecto 8864: "Investigación de la Biodiversidad y los servicios ecosistémicos para la gestión y el fortalecimiento de las capacidades descubriendo los ecosistemas estratégicos para el fortalecimiento de la gobernanza en el departamento de Santander" y Programa Bio-Reto XXI 15:50. Proyecto 1: "Inventario de la diversidad biológica en una región del sur de Bolívar - Colombia", por su continuo apoyo.

\section{RESUMEN}

Páramos Neotropicales como unidades biogeográficas. Introducción: El Páramo se ha definido desde diversos acercamientos, teniendo en cuenta factores de fácil reconocimiento o medición. A nivel biogeográfico se ha evaluado con métodos ampliamente criticados para la identificación de áreas de endemismo. El análisis de endemicidad, pese a su importancia y amplio reconocimiento, no se ha utilizado como herramienta para evaluar el Páramo. Objetivo: Determinar si los páramos neotropicales es una o varias unidades biogeográficas. Métodos: Incluimos registros de Aves, Amphibia, Mammalia, Reptilia, Marchantiophyta y Spermatophyta, para los que encontramos 7025 especies con 193250 presencias viables obtenidas desde GBIF (Global Biodiversity Information Facility) [a septiembre de 2018]. Usamos cada grupo taxonómico como una partición independiente, y generamos particiones adicionales como plantas totales (Plantas-T: Marchantiophyta + Spermatophyta), animales totales (Animales-T: Aves + Amphibia + Mammalia + Reptilia) y evidencia total (Plantas-T + Animales-T). Utilizamos el criterio de optimalidad para identificar áreas de endemismo. Realizamos el análisis usando dos tamaños de cuadrícula 0.5 y $0.25^{\circ}$. Con las áreas obtenidas, calculamos la intersección con los polígonos que representan las definiciones de páramo generadas por otros autores. Resultados: Con los dos tamaños de cuadrícula identificamos áreas de endemismo en diferentes sectores; sin embargo, el tamaño de $0.25^{\circ}$ nos permitió mayor resolución al identificar los sectores en alta montaña. Estos sectores corresponden a ocho zonas que denominamos subprovincias: Santa Marta-Perijá, Mérida, Santanderes-Boyacá, Cundinamarca, Cordillera CentralOccidental, Norte de Ecuador, Centro-Sur de Ecuador y Talamanca, las cuales fueron congruentes entre un 4 y un $66 \%$ con las definiciones previas. Conclusiones: Páramo se ha planteado como una sola unidad biogeográfica; sin embargo, dado nuestros análisis, lo identificamos como ocho subprovincias biogeográficas, congruentes con estudios previamente publicados.

Palabras clave: área de endemismo, NDM/VNDM, análisis de endemicidad, subprovincia biogeográfica, Páramo.

\section{REFERENCIAS}

Aagesen, L., Szumik, C., \& Goloboff, P. (2013). Consensus in the search for areas of endemism. Journal of Biogeography, 40(11), 2011-2016.

Aagesen, L., Szumik, C., Zuloaga, F.O., \& Morrone, J. (2009). Quantitative biogeography in the South America highlands-recognizing the Altoandina, Puna and Prepuna through the study of Poaceae. Cladistics, 25(3), 295-310.

Alvez-Valles, C.M., Balslev, H., Carvalho, F.A., GarciaVillacorta, R., Grandez, C., \& Neto, L.M. (2018). 
Endemism and conservation of Amazon palms. Biodiversity and Conservation, 27(3), 765-784.

Antonelli, A., Condamine, F.L., Hettling, H., Nilsson, K., Nilsson, R.H., Oxelman, B., . . . Silvestro, D. (2014). SUPERSMART: ecology and evolution in the era of big data. PeerJ PrePrints, 2:e501v1.

Benham, P.M., Cuervo, A.M., McGuire, J.A., \& Witt, C.C. (2015). Biogeography of the Andean metaltail hummingbirds: contrasting evolutionary histories of tree line and habitat-generalist clades. Journal of Biogeography, 42(4), 763-777.

Bivand, R., Rundel, C., Pebesma, E., Stuetz, R., \& Hufthammer, K. (2016). Rgeos: interface to geometry engine-open source (GEOS) [Software]. Disponible en https://CRAN.R-project.org/package=rgeos

Brack, A., \& Mendiola, C. (2000). Ecología del Perú. Lima: Editorial Bruño.

Buytaert, W., Iniguez, V., \& De Bievre, B. (2007). The effects of afforestation and cultivation on water yield in the andean páramo. Forest Ecology and Management, 251(1-2), 22-30.

Cabrera, A., \& Willink, A. (1980). Biogeografía de América Latina. Washington, DC: Organización de los Estados Americanos.

Casagranda, D., Roig-Juñent, S., \& Szumik, C. (2009). Endemismo a diferentes escalas espaciales: un ejemplo con Carabidae (Coleoptera: Insecta) de América del Sur austral. Revista Chilena de Historia Natural, 82(1), 17-42.

Casagranda, M.D., Taher, L., \& Szumik, C.A. (2012). Endemicity analysis, parsimony and biotic elements: a formal comparison using hypothetical distributions. Cladistics, 28(6), 645-654.

Chapman, A. (2005). Uses of the primary species occurrence data, version 1.0. Report for the global biodiversity information facility, Copenhagen.

Cleef, A., \& Chaverri, A. (1992). Phytogeography of the páramo flora of Cordillera de Talamanca, Costa Rica. In H. Balslev \& J. Luteyn (Eds.), Páramo: An Andean ecosystem under human influence (pp. 45-60). London: Academic Press.

Cortés-Duque, J., \& Sarmiento Pinzón, C.E. (2017). Visión socioecosistémica de los páramos y la alta montaña colombiana. Memorias del proceso de definición de criterios para la delimitación de páramos. Bogotá: Instituto de Investigación de Recursos Biológicos Alexander von Humboldt.

Cuatrecasas, J. (1958). Aspectos de la vegetación natural de Colombia. Revista de la Academia Colombiana de Ciencias Exactas, Físicas y Naturales, 10(40), 221-268.
De Mera, A.G., Vega, I.S., Quino, J.M., Perea, E.L., De La Cruz, J.C., \& Orellana, J.A.V. (2017). La vegetación del norte del Perú: De los bosques a la Jalca en Cajamarca. Acta Botánica Malacitana, 40(1), 157-190.

Deo, A.J., \& De Salle, R. (2006). Nested areas of endemism analysis. Journal of Biogeography, 33(9), 1511-1526.

Diazgranados, M., \& Barber, J.C. (2017). Geography shapes the phylogeny of frailejones (Espeletiinae Cuatrec., Asteraceae): a remarkable example of recent rapid radiation in sky islands. PeerJ, 5,e2968.

Dos Santos, D.A., Fernández, H.R., Cuezzo, M.G., \& Dominguez, E. (2008). Sympatry inference and network analysis in biogeography. Systematic Biology, 57(3), 432-448.

Ebach, M.C., Morrone, J.J., Parenti, L.R., \& Viloria, A.L. (2008). International code of area nomenclature. Journal of Biogeography, 35(7), 1153-1157.

Escalante, T. (2009). Un ensayo sobre regionalización biogeográfica. Revista Mexicana de Biodiversidad, $80(2), 551-560$.

Escalante, T. (2011). De cómo el análisis de parsimonia de endemismos (PAE) tampoco explica la selección natural. Revista Mexicana de Biodiversidad, 82(3), 1057-1059.

Escalante, T. (2015). Parsimony analysis of endemicity and analysis of endemicity: A fair comparison. Systematics and Biodiversity, 13(5), 413-418.

Escalante, T., Rodríguez-Tapia, G., Szumik, C., Morrone, J.J., \& Rivas, M. (2010). Delimitation of the Nearctic region according to mammalian distributional patterns. Journal of Mammalogy, 91(6), 1381-1388.

Flantua, SG., \& Hooghiemstra, H. (2018). Historical connectivity and mountain biodiversity. In C. Hoorn, A. Perrigo, \& A. Antonelli (Eds.), Mountains, climate and biodiversity (pp. 171-185). Hoboken, NJ: John Wiley \& Sons.

Flantua, S.G., O’dea, A., Onstein, R.E., Giraldo, C., \& Hooghiemstra, H. (2019). The flickering connectivity system of the north Andean páramos. Journal of Biogeography, 46(8), 1808-1825.

García-Roselló, E., Guisande, C., Manjarrés-Hernández, A., González-Dacosta, J., Heine, J., Pelayo-Villamil, P., . . . Granado-Lorencio, C. (2015). Can we derive macroecological patterns from primary Global Biodiversity Information Facility data? Global Ecology and Biogeography, 24(3), 335-347.

Garzón-Orduña, I.J., Miranda-Esquivel, D.R., \& Donato, M. (2008). Parsimony analysis of endemicity describes but does not explain: an illustrated critique. Journal of Biogeography, 35(5), 903-913. 
Giokas, S., \& Sfenthourakis, S. (2008). An improved method for the identification of areas of endemism using species co-occurrences. Journal of Biogeography, 35(5), 893-902.

Goloboff, P. (2005). Programs for identification of areas of endemism [Software]. Disponible en https://www. lillo.org.ar/phylogeny/endemism

Gonzalez-Duran, G.A. (2016). A new small frog species of the genus Pristimantis (Anura: Craugastoridae) from the northern paramos of Colombia. Zootaxa, 4066(4), 421-437.

Guhl, E. (1982). Los páramos circundantes de la sabana de Bogotá. Bogotá: Jardín Botánico de Bogotá José Celestino Mutis.

Harold, A.S., \& Mooi, R.D. (1994). Areas of endemism: definition and recognition criteria. Systematic Biology, 43(2), 261-266.

Hazzi, N.A., Moreno, J.S., Ortiz-Movliav, C., \& Palacio, R.D. (2018). Biogeographic regions and events of isolation and diversification of the endemic biota of the tropical Andes. Proceedings of the National Academy of Sciences, 115(31), 7985-7990.

Hernández-Camacho, J., Hurtado, A., Ortiz, R., \& Walschburger, T. (1992). Unidades biogeográficas de Colombia. En G. Halffter (Ed.), La diversidad biológica de Iberoamérica (pp. 105-151). Xalapa, México: Instituto de Ecología, A.C.

Hofstede, R., Calles, J., López, V., Polanco, R., Torres, F., Ulloa, J., . . . Cerra, M. (2014). Los Páramos Andinos ¿Qué sabemos? Estado de conocimiento sobre el impacto del cambio climático en el ecosistema páramo. Quito: UICN.

Hofstede, R., Segarra, P., \& Vásconez, P.M. (2003). Los páramos del mundo: Proyecto Atlas Mundial de los Páramos. Quito: Ecociencia.

Jiménez-Rivillas, C., García, J.J., Quijano-Abril, M.A., Daza, J.M., \& Morrone, J.J. (2018). A new biogeographical regionalisation of the Páramo biogeographic province. Australian Systematic Botany, 31(4), 296-310.

Jørgensen, P., \& Ulloa, C. (1994). Seed plants of the high Andes of Ecuador: A checklist. Aarhus, Denmark: Aarhus University Press.

Kells, S.S., \& Worswick, C. (1997). An introduction to the IBIS database. Parkville: Melbourne Institute of Applied Economic and Social Research.

Linder, H. (2001). On areas of endemism, with an example from the African Restionaceae. Systematic Biology, 50(6), 892-912.

Londoño, C., Cleef, A., \& Madriñán, S. (2014). Angiosperm flora and biogeography of the páramo region of Colombia, Northern Andes. Flora - Morphology, Distribution, Functional Ecology of Plants, 209(2), 81-87.

Lowe, S., Browne, M., Boudjelas, S., \& DePoorter, M. (2000). 100 of the world's worst invasive alien species: a selection from the global invasive species database. Auckland, New Zealand: Invasive Species Specialist Group Auckland.

Luteyn, J.L. (1999). Páramos: A Checklist of Plant Diversity, Geographical Distribution, and Botanical Literature. New York, NY: The New York Botanical Garden Press.

Luteyn, J.L., \& Balslev, H. (1992). Páramo: an Andean ecosystem under human influence. London, UK: Academic Press.

Madriñán, S., Cortés, A.J., \& Richardson, J.E. (2013). Páramo is the world's fastest evolving and coolest biodiversity hotspot. Frontiers in Genetics, 4, 192.

Maldonado, C., Molina, C.I., Zizka, A., Persson, C., Taylor, C.M., Albán, J., . . . Antonelli, A. (2015). Estimating species diversity and distribution in the era of Big Data: to what extent can we trust public databases? Global Ecology and Biogeography, 24(8), 973-984.

Martínez-Hernández, F., Mendoza-Fernández, A., PérezGarcía, F., Martínez-Nieto, M., Garrido-Becerra, J., Salmerón-Sánchez, E., . . . Mota, J. (2015). Areas of endemism as a conservation criterion for Iberian gypsophilous flora: a multi-scale test using the NDM/ VNDM program. Plant Biosystems, 149(3), 483-493.

Mast, A.R., \& Nyffeler, R. (2003). Using a null model to recognize significant co-occurrence prior to identifying candidate areas of endemism. Systematic Biology, 52(2),271-280.

Monasterio, M. (1980). Los páramos andinos como región natural. Características biogeográficas generales y afinidades con otras regiones andinas. In M. Monasterio (Ed.), Estudios ecológicos en los páramos andinos (pp. 15-27). Bogotá: Ediciones Universidad de Los Andes.

Morales-Rivas, M., Otero Garcia, J., Van der Hammen, T., Torres Perdigón, A., Cadena Vargas, C.E., Pedraza Peñaloza, C.A., . . . Olaya Ospina, É. (2007). Atlas de páramos de Colombia. Bogotá: Instituto de Investigación de Recursos Biológicos Alexander von Humboldt.

Morrone, J.J. (1994). On the identification of areas of endemism. Systematic Biology, 43(3), 438-441.

Morrone, J.J. (2001). Biogeografia de América Latina y el Caribe. Zaragoza, España: Sociedad Entomológica Aragonesa.

Morrone, J.J. (2014). Biogeographical regionalisation of the neotropical region. Zootaxa, 3782(1), 1-110. 
Morrone, J.J. (2017). Neotropical Biogeography: Regionalization and Evolution. Boca Raton, FL: CRC Press.

Noguera-Urbano, E. (2017). El endemismo: diferenciación del término, métodos y aplicaciones. Acta Zoológica Mexicana, 33(1),89-107.

Oliveira, U., Brescovit, A.D., \& Santos, A. (2015). Delimiting Areas of Endemism through Kernel Interpolation. PLOS ONE, 10(1), e0116673.

Platnick, N. (1991). On areas of endemism. Australian Systematic Botany, 4(1), 11-12.

QGIS Development Team (2019). QGIS Geographic Information System [computer software]. Chicago: Open Source Geospatial Foundation Project.

Quintero, E., Ribas, C.C., \& Cracraft, J. (2012). The Andean Hapalopsittaca parrots (Psittacidae, Aves): an example of montane-tropical lowland vicariance. Zoologica Scripta, 42(1), 28-43.

R Core Team (2019). R: A language and environment for statistical computing. R Foundation for Statistical Computing, Vienna, Austria. [software]. Disponible en https://www.R-project.org/

RAE (2014). Diccionario de la lengua española (23 ${ }^{\text {ra }}$ ed.). Madrid, España: Real Academia Española.

Rangel, J.O. (2000). Colombia. Diversidad biótica III: La región de vida paramuna. Bogotá: Universidad Nacional de Colombia.

Romero-Alarcon, L.V., \& Miranda-Esquivel, D.R. (2015). biogeoclean: An $\mathrm{R}$ package to cleaning geographical data from electronic biodatabases (Version 3.4.0) [Software]. Disponible en https://github.com/ alarconvv/biogeoclean

Romero-Alarcon, L.V. (2016). Patrón panbiogeográfico de la Provincia Chocó (Tesis de Pregrado). Universidad Industrial de Santander, Santander, Colombia.

Rosen, B.R., \& Smith, A.B. (1988). Tectonics from fossils? Analysis of reef-coral and sea-urchin distributions from late Cretaceous to Recent, using a new method. Geological Society, London, Special Publications, 37(1), 275-306.

Sánchez, I.E., Garcia-Cruz, J., Espejo-Serna, A., \& LopezOrtega, G. (2019). Identification of areas of endemism in the Mexican cloud forests based on the distribution of endemic epiphytic bromeliads and orchids. Phytotaxa, 397(2), 129-145.
Sarmiento, C., Cadena, C., Sarmiento, M., Zapata, J., \& León, O. (2013). Aportes a la conservación estratégica de los páramos de Colombia: actualización de la cartografía de los complejos de páramo a escala 1:100.000. Bogotá: Instituto de Investigación de Recursos Biológicos Alexander von Humboldt.

Shaw, C.A. (2004). ITIS (The Integrated Taxonomic Information System) [On-line database]. Retrieved from https://www.itis.gov

Szumik, C., Aagesen, L., Casagranda, D., Arzamendia, V., Baldo, D., Claps, L.E., . . . Giraudo, A. (2012). Detecting areas of endemism with a taxonomically diverse data set: plants, mammals, reptiles, amphibians, birds, and insects from Argentina. Cladistics, 28(3), 317-329.

Szumik, C.A., Cuezzo, F., Goloboff, P.A., \& Chalup, A.E. (2002). An optimality criterion to determine areas of endemism. Systematic Biology, 51(5), 806-816.

Szumik, C.A., \& Goloboff, P.A. (2004). Areas of endemism: an improved optimality criterion. Systematic Biology, 53(6), 968-977.

Telenius, A. (2011). Biodiversity information goes public: GBIF at your service. Nordic Journal of Botany, 29(3), 378-381.

Tropicos (2018). Missouri Botanical Garden [On-line database]. Disponible en https://www.tropicos.org

Van der Hammen, T. (1974). The pleistocene changes of vegetation and climate in tropical South America. Journal of Biogeography, 1(1), 3-26.

Van der Hammen, T. (1997). Ecosistemas terrestres-páramos. En M. Chavez \& N. Arango (Eds.), Informe Nacional sobre el Estado de la Biodiversidad (pp. 10-37). Bogotá: Instituto de Investigación de Recursos Biológicos Alexander von Humboldt.

Van der Hammen, T., \& Cleef, A.M. (1986). Development of the high andean páramo flora and vegetation. In F. Vuilleumier \& M. Monasterio (Eds.), High altitude tropical biogeography (pp. 153-201). Oxford: Oxford University Press.

Van der Hammen, T., \& González, E. (1963). Historia de clima y vegetación del Pleistoceno Superior y del Holoceno de la Sabana de Bogotá. Boletín Geológico, 11(1-3), 189-266.

Vargas, O., \& Pedraza, P. (2004). Parque Nacional Natural Chingaza. Bogotá: Universidad Nacional de Colombia. 
Vuilleumier, F., \& Monasterio, M. (1986). High altitude tropical biogeography. Oxford: Oxford University Press.

Wijninga, V. (1996). Palynology and paleobotany of Neogene sediments from the high plain of Bogotá (Colombia): evolution of the Andean flora from an ecological perspective ( $\mathrm{PhD}$ Thesis). University of Amsterdam, Netherlands.

Zhuang, H., Yago, M., Settele, J., Li, X., Ueshima, R., Grishin, N.V., \& Wang, M. (2018). Species richness of Eurasian Zephyrus hairstreaks (Lepidoptera:
Lycaenidae: Theclini) with implications on historical biogeography: An NDM/VNDM approach. PLOS ONE, 13(1), e0191049.

Zizka, A., Silvestro, D., Andermann, T., Azevedo, J., DuarteRitter, C., Edler, D., . . . Antonelli, A. (2019). CoordinateCleaner: Standardized cleaning of occurrence records from biological collection databases. Methods in Ecology and Evolution, 10(5), 744-751.

Zunino, M., \& Zullini, A. (2003). Biogeografia: la dimensión espacial de la evolución. México, DF: Fondo de Cultura Económica.

See Digital Appendix at: / Ver Apéndice digital en:

revistas.ucr.ac.cr 